\section{Equidad de género en las políticas de reforma del sector de la salud de América Latina y el Caribe}

\author{
Elsa Gómez Gómez
}

Palabras clave: reforma del sector de la salud, políticas de salud, equidad, género.

Programa Mujer, Salud y Desarrollo. División de Salud y Desarrollo Humano. Organización Panamericana de la Salud. Dirección postal: 525 Twenty-third St., N.W., Washington, D.C. 20037, Estados Unidos de América.
La Organización Panamericana de la Salud (OPS), mediante su Programa Mujer, Salud y Desarrollo de la División de Salud y Desarrollo Humano, viene impulsando desde hace año y medio un proyecto encaminado a promover la equidad de género en las reformas del sector de la salud de la Región. Esta iniciativa se originó durante una consulta regional con expertos en materia de género y política, convocada por la OPS en octubre de 1998, la cual cristalizó en un plan de trabajo regional que fue aprobado en febrero de 1999 por el Subcomité Especial sobre Mujer, Salud y Desarrollo del Comité Ejecutivo de la OPS. La primera etapa del proyecto recibió financiamiento de las Fundaciones Ford y Rockefeller en el año 2000 y está ejecutándose en Chile y Perú, paralelamente con actividades a escala regional.

El eje central del proyecto es la producción y utilización de la información como instrumento de cambio en favor de la equidad de género en salud. Las desigualdades que constituyen el blanco de las acciones del proyecto son las disparidades innecesarias, evitables e injustas entre hombres y mujeres en el estado de salud, el acceso a la atención de salud y la participación en la toma de decisiones dentro del sistema de salud. Un énfasis particular, pero no exclusivo, se ha asignado a las desigualdades que ocurren como consecuencia de respuestas institucionales inapropiadas a la luz de las necesidades en salud sexual y reproductiva.

El acento en el género no oscurece en forma alguna la importancia de otras desigualdades de poder. Por el contrario, el enfoque de este proyecto subraya el imperativo de plantear el análisis y la acción en torno al género dentro del marco de las desigualdades de clase, raza, edad y región geográfica, categorías en las que la inequidad de género se expresa de formas diferentes.

\section{MARCO INSTITUCIONAL}

Este proyecto se fundamenta en los dos principios rectores de la OPS: la búsqueda de la equidad, mandato central y distintivo de la Organización, y el panamericanismo, valor que promueve la participación activa y conjunta de los países en la formulación de estrategias para el logro de la salud para todos.

La reducción de las inequidades en el campo de la salud y el desarrollo humano se ha declarado 
el objetivo fundamental de la cooperación técnica de la OPS y, dentro de este contexto, "la incorporación de una perspectiva de género en las políticas y estrategias sanitarias" ha sido identificada como un valor clave. En la misma línea, las Orientaciones Estratégicas y Programáticas de la OPS estipulan que la cooperación técnica fomentará el "desarrollo de la capacidad de usar la perspectiva de género como una herramienta para analizar la repercusión de la globalización en el proceso de desarrollo y en las políticas estructurales, macroeconómicas y sociales, haciendo hincapié en su relación con la salud" (1).

\section{JUSTIFICACIÓN Y OBJETIVOS}

La equidad de género se reconoce cada vez más como elemento esencial para el desarrollo sostenible y, en particular, para el desarrollo de la salud pública. La consideración de las diferencias entre hombres y mujeres, no solo en materia de necesidades, sino también de contribuciones y de acceso y control sobre los recursos, debería constituir un elemento esencial de las políticas sectoriales. Lamentablemente, la dimensión del género permanece marginada del debate público sobre la equidad, sin integrarse dentro de la corriente central de la formulación y evaluación de las políticas de reforma del sector de la salud.

Sólidas pruebas - aunque todavía un tanto fragmentarias- sugieren que las actuales reformas del sector de la salud están contribuyendo con demasiada frecuencia a exacerbar inequidades de género, tanto en el ámbito de la utilización como de la provisión de atención. En efecto, las repercusiones adversas para la equidad en salud de medidas tales como la recuperación de costos en el sector público, la privatización del financiamiento de la atención, la reducción del gasto público en salud e, incluso, ciertas estrategias de descentralización, tienden a afectar desproporcionadamente a las mujeres, particularmente las de menos recursos económicos, por las siguientes razones:

- Dadas su función reproductiva y mayor longevidad, las mujeres tienen mayor necesidad de servicios de salud que los hombres a lo largo de la vida.

- Las mujeres constituyen una mayoría de los pobres y, por razón de su participación desventajosa en el mercado de trabajo, tienen menos acceso que los hombres a los recursos económicos y laborales que permiten la "compra" de servicios y la participación directa en sistemas de aseguramiento de salud.

- Por razón del "riesgo de embarazo", el acceso de las mujeres en edades reproductivas a planes de aseguramiento de salud frecuentemente implica cuotas económicas más altas que para los hombres. Adicionalmente, por su mayor necesidad de servicios, las mujeres tienen que pagar más que los hombres en gastos de bolsillo para la salud, gastos que por su carácter regresivo resultan ser los menos equitativos.

- Pese a la función protagónica de las mujeres en la gestión de la salud - ya sea en el ámbito institucional o en el seno de la familia y la comunidad-, estas permanecen subrepresentadas en las estructuras de poder local, nacional y sectorial que definen prioridades y asignan recursos. La poca participación de las mujeres en la toma de decisiones hace que con frecuencia sus necesidades no lleguen a considerarse prioritarias ni a recibir asignaciones apropiadas de los recursos disponibles.

- Los recortes en los servicios de salud trasladan a los hogares parte de la atención que debe proveer el Estado, con lo que aumenta la carga de cuidados que asumen predominantemente las mujeres en el seno de la familia y la comunidad.

El diseño de este proyecto responde a la necesidad de llenar el vacío existente en el debate y la formulación de políticas en favor de la equidad en salud, mediante el fomento de acciones coordinadas entre los gobiernos y la sociedad civil en torno a los siguientes objetivos:

- documentar y analizar las inequidades de género en el ámbito de la salud, y la asociación de las mismas con las políticas de salud

- comunicar esta información estratégicamente a actores clave con el fin, por un lado, de sensibilizar e informar a los gestores de políticas $\mathrm{y}$, por el otro, de potenciar la acción de los grupos de la sociedad civil que abogan por la equidad de género

- fomentar el diálogo y la formación de coaliciones entre actores del gobierno, el sector privado y la sociedad civil, para incidir en la formulación de políticas y la rendición de cuentas

- institucionalizar la participación de actores clave de distintos sectores del gobierno y la sociedad civil en la formulación y fiscalización de las políticas en favor de la equidad de género.

\section{ESTRATEGIAS Y ACTIVIDADES}

El proyecto Equidad de género en las políticas de reforma del sector de salud se está desarrollando a escala regional e, inicialmente, en dos países piloto: Chile y Perú. En Chile el proyecto comenzó en febrero de 2001 y en Perú apenas está iniciándose. 
A escala regional, el proyecto se concentra en las siguientes tareas:

- coordinar la producción, sistematización y diseminación de la información pertinente

- construir y validar herramientas conceptuales y metodológicas que sirvan de punto de partida para el trabajo en los países, con respecto a la medición, el análisis y el monitoreo de inequidades de género en la situación de salud y en las políticas de reforma

- promoción de trabajos de colaboración en favor de la equidad de género en la reforma del sector de la salud entre: a) actores relacionados con este problema en la Región, b) equipos nacionales que desarrollan el proyecto $\mathrm{y}, \mathrm{c}$ ) unidades técnicas de la OPS vinculadas directamente al área de políticas.

A escala nacional, el proyecto fomenta el diálogo y facilita la concertación de acciones entre actores clave del gobierno y la sociedad civil alrededor de los siguientes objetivos:

- Fortalecimiento de la capacidad de análisis en sectores del gobierno, y de abogacía en grupos de la sociedad civil

- Elaboración de diagnósticos en aspectos prioritarios de la utilización y provisión de servicios. Dentro de este ámbito diagnóstico se destacan como novedosos: a) la medición de la contribución de los hogares - y del trabajo no remunerado de las mujeres - a la producción de la salud, con énfasis en el sistema de Cuentas Nacionales en Salud, y la fiscalización de presupuestos, y b) la evaluación de la ejecución presupuestal desde la perspectiva del género.

- Discusión conjunta de las propuestas oficiales de reformas en foros nacionales y provinciales. En Chile, estos foros han congregado a representantes del Ministerio de Salud, Comisiones de Reforma, Ministerio de la Mujer, Ministerio de Hacienda, legisladores, universidades, gremios y organizaciones de mujeres.

- Formulación de medidas que corrijan las inequidades de género en cuestiones prioritarias definidas participativamente por actores del gobierno y la sociedad civil

- Creación de mecanismos para institucionalizar y vigilar la ejecución de tales medidas, incluido el mejoramiento de los sistemas de información.

\section{RESULTADOS ESPERADOS}

$\mathrm{Al}$ cabo de tres años se espera que el proyecto haya catalizado cambios en los siguientes ámbitos:
1. En el campo de la producción de información y conocimientos, se espera lograr:

- la definición de un conjunto de indicadores básicos para la identificación, el análisis y la vigilancia de las desigualdades de género en el área de la salud

- la sistematización y análisis de información clave relacionada con la medición de desigualdades de género en el ámbito de la salud, y con la asociación entre tales desigualdades y las políticas de salud.

2. En el tema de la abogacía y difusión de información, el proyecto espera facilitar:

- el acceso fácil a la información generada o sistematizada por el proyecto para uso de políticos, planificadores, investigadores y grupos de la sociedad civil que aboguen por la equidad en sentido amplio y, en particular, la equidad de género

- el fortalecimiento de la capacidad de los ministerios de salud y de asuntos de la mujer, de los institutos de estadística, de grupos parlamentarios y de otros organismos del Estado relacionados con este problema, para integrar criterios de equidad de género en las políticas de reforma del sector de la salud y en los sistemas de información, vigilancia y rendición de cuentas en salud

- el fortalecimiento de la capacidad de abogacía y de organización de los grupos de mujeres y otros grupos defensores de la equidad, para participar en la formulación y la fiscalización de las políticas de reforma del sector de la salud

- la formulación de propuestas intersectoriales de políticas en favor de la equidad de género que respondan a prioridades definidas participativamente por los actores interesados.

3. En el plano de la institucionalización, el proyecto habría inspirado la creación y apropiación -o por lo menos, la identificación- de mecanismos intersectoriales que permitan articular los actores clave del gobierno y la sociedad civil para la formulación y vigilancia de políticas. Dentro de estos mecanismos, figurarían de manera prominente:

- un sistema de información que incorpore indicadores de género y cuyos elementos reflejen la interacción entre productores y usuarios de la información

- un sistema de monitoreo de políticas que tome en cuenta la problemática del género, facilite la rendición de cuentas y en el que concurran actores clave del gobierno y la sociedad civil.

El desafío es triple. Primero, se busca que la producción de información se desarrolle con el con- 
curso de productores y usuarios de manera que garantice su pertinencia política y se institucionalice en organismos del Estado. Segundo, se aspira a que esta información se traduzca efectivamente en políticas en favor de la equidad de género, cuya formulación involucre una participación ciudadana que garantice que las necesidades de género se tomen en cuenta. Y tercero, que la ejecución y la sostenibilidad de estas políticas se apoyen en una cultura cívica que vigile su cumplimiento y exija la rendición de cuentas.

\section{SYNOPSIS}

\section{Gender equity in health sector reform policies in Latin America and the Caribbean}

Gender equity is increasingly being acknowledged as an essential aspect of sustainable development and more speci- fically, of health development. The Pan American Health Organization's Program for Women, Health, and Development has been piloting for a year now a project known as Equidad de género en las políticas de reforma del sector de salud, whose objective is to promote gender equity in the health sector reform efforts in the Region. The first stage of the project is being conducted in Chile and Peru, along with some activities throughout the Region. The core of the project is the production and use of information as a tool for introducing changes geared toward achieving greater gender equity in health, particularly in connection with malefemale disparities that are unnecessary, avoidable, and unfair in health status, access to health care, and participation in decision-making within the health system. We expect that in three years the project will have brought about changes in the production of information and knowledge, advocacy, and information dissemination, as well as in the development, appropriation, and identification of intersectoral mechanisms that will make it possible for key figures in government and civil society to work together in setting and surveying policy on gender equity in health.

\section{REFERENCIA}

1. Pan American Health Organization. Strategic and programmatic orientations for the Pan American Sanitary Bureau, 19992002. 25th Pan American Sanitary Confe- rence, Washington, D.C., September 1998 P. 29. (Documento CSP25/8). 